\title{
Der Wunsch nach vorzeitigem Lebensende
}

? Dr. Simon, was schätzen Sie: Wie viele Patienten äußern in Deutschland den Wunsch nach einem vorzeitigen Sterben?

PD Dr. Alfred Simon: Studien aus Deutschland und anderen Ländern zeigen, dass etwa jeder siebte bis zehnte Patient mit einer schweren, zum Tode führenden Krankheit zumindest einmal im Laufe seiner Erkrankung einen solchen Wunsch äußert.

?... und bei wie vielen Patienten ist die Entscheidung unumstößlich?

Simon: Hierzu ist die Studienlage nicht eindeutig. Während mehrere Studien gezeigt haben, dass Lebensund Todeswunsch von schwerkranken Patienten sehr schwanken, ergab eine aktuelle Studie aus Frankreich, dass bei einem Drittel der Patienten mit Sterbewunsch der Wunsch trotz palliativer Versorgungsangebote Bestand hatte.

? Welche Hauptgründe für ihren Wunsch nennen die Patienten?

Simon: Angst vor der Zukunft, Angst vor Verlust an Autonomie und Kontrolle, keine Belastung für andere sein zu wollen, das Wahrnehmen der eigenen Situation als aussichts- und hoffnungslos: Das sind einige der Gründe.

? Ist die Palliativmedizin nicht in der Lage, den Wunsch überflüssig zu machen?

Simon: Nicht in allen Fällen. Denn auch bei guter palliativer Versorgung wird es Patienten geben, deren
Leiden nicht angemessen gelindert werden kann oder die einen schnellen Tod den palliativen Angeboten vorziehen.

? Sie sagten: Der Wunsch nach Sterbehilfe ist immer der Wunsch nach Hilfe. Was meinen Sie damit genau?

Simon: Nicht jeder Wunsch nach Sterbehilfe ist der Wunsch nach Hilfe zu einem vorzeitigen Lebensende. Aber in jedem Fall ist er der Wunsch nach Hilfe in einer vom Patienten als unerträglich oder bedrohlich erlebten Situation.

? Wie groß ist die Gefahr, dass sich Patienten nicht frei entscheiden, sondern unter moralischem Zwang stehen?

Simon: Patienten werden sich dann gezwungen sehen, nach Sterbehilfe zu fragen, wenn es für sie keine anderen Alternativen gibt. Also sollten wir uns fragen, welche möglichen Alternativen wir ihnen anbieten können. Wenn der Wunsch nach Sterbehilfe dann trotz dieser Alternativen fortbesteht, ist die Wahrscheinlichkeit groß, dass es sich bei dem Sterbehilfewunsch um eine freie Patientenentscheidung handelt.

? Wie sehen Sie die künftige Entwicklung in Deutschland im Umgang mit Patienten, die den Wunsch nach Sterbehilfe haben?

Simon: Ich denke, dass diese Entwicklung im Moment ziemlich offen ist.

Depression ist eine bedeutende Einflussvariable Stephanie Stiel, Universitätsklinikum Erlangen, und ihre Kollegen vom Universitätsklinikum der RWTH Aachen erinnern in einem Beitrag in der Fachzeitschrift „Der Schmerz“ daran, dass seit mehr als zwei Dekaden untersucht wird, inwiefern klinisch bedeutsame psychische Symptome wie Angst und Depression mit dem Patientenwunsch, vorzeitig $\mathrm{zu}$ sterben, korrelieren. Ihrer Ansicht nach zeichnen sich Depressionen als bedeutende Einflussvariable auf den Sterbewunsch ab.

Depressive Verstimmungen wirken sich auch auf die Autonomie der Patienten aus. Julian Bleek vom Vivantes Klinikum in Berlin legte im vergangenen Jahr in „Ethik in der Medizin“ dar, dass depressive Störungen empirischen Studien zufolge die Selbstbestimmungsfähigkeit so sehr beeinträchtigen können, „dass von einer selbstbestimmten Entscheidung nicht mehr gesprochen werden kann“. Er

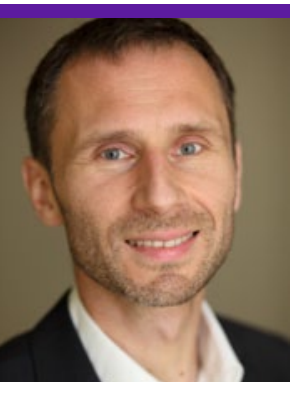

PD Dr. Alfred Simon, Akademie für Ethik in der Medizin e.V., Göttingen

, genuber Angehörigen oder glaubte, diesen zur Last 48(3):368-76.]. Tatsächlich ließen sich aber nur bei 3,7\% die Schmerzen nicht ausreichend wirksam unterdrücken. Fast jeder Dritte galt als ängstlich-depressiv. 